\title{
Investigating the Impact of Weather Parameters on Signal Strength of Satellite Dish in Enugu Metropolis
}

\author{
Chima Abraham Iheanyichukwu, Onyia Augustine Ike, Chikeleze Praise Chukwuemeka
}

Department of Industrial Physics, Enugu State University of Science and Technology, Enugu, Nigeria

\section{Email address:}

abraham.chima@esut.edu.ng (C. A. Iheanyichukwu)

\section{To cite this article:}

Chima Abraham Iheanyichukwu, Onyia Augustine Ike, Chikeleze Praise Chukwuemeka. Investigating the Impact of Weather Parameters on Signal Strength of Satellite Dish in Enugu Metropolis. International Journal of Astrophysics and Space Science.

Vol. 8, No. 2, 2020, pp. 11-15. doi: 10.11648/j.ijass.20200802.11

Received: July 3, 2020; Accepted: July 15, 2020; Published: August 4, 2020

\begin{abstract}
This research evaluates the impact of weather parameter (temperature, pressure, humidity and wind speed) on satellite dishes and how it affects the quality of reception it gives using the data obtained from the Automated Weather Station and Signal Strength in Enugu State University of Science and Technology. The atmospheric components and signal strength were measured every two minutes daily in different months, on different days, and at a different time of the day from the station for period of nine months. The average results of these measurements were then taken and the data obtained from the measurements were tabulated and subsequently plotted in a graph to ascertain the variation in signal strength triggered by change in temperature, humidity, pressure and wind speed. It was observed from this research that the rise in atmospheric temperature, humidity and pressure will lead to a drop in strength of the signal generated by this station and vice versa. This indicated that signal strength is inversely proportional to atmospheric temperature, pressure and humidity; provided that for any of the giving components, others were observed constant, including the wind speed and direction which affects the positioning of satellite dishes. The correlation of the signal strength and atmospheric temperature, pressure and humidity were respectively $\mathrm{r}=-0.93,-0.97$ and -0.92 . It was observed that the atmospheric temperature, humidity, pressure and wind speed are mathematically inversely related.
\end{abstract}

Keywords: Temperature, Pressure, Humidity, Signal Strength

\section{Introduction}

Satellite television is a service that delivers television programming to viewers by relaying it from a communications satellite orbiting the earth directly to the viewer's locations [1]. The satellite collects its information or receives radio-frequency signals, uplinked from the earth station, it then amplifies the signals, changes the frequency and send them on a downlink frequency for transmission to one or more satellite dishes on earth. The dish connection of the satellite has to be direct since the signal travel direct to the satellite for transmission. Any alteration of wrong direction of the dish can lead to lose of signal in transmission. Satellites are designed to send and receive data from other satellites or earth stations. This data may carry voice, audio, video, or other information, and it is the basis for satellite television and certain kinds of internet access cell phone users [2].
Satellite dishes are used to receive television broadcast signals directly through the air and transmit it to the accompanying TV set. In some places, especially rural areas, TV antennas and dishes are the only ways to get quality television programming. When the signal reaches the viewer's house, it is captured by the satellite dish. A satellite dish is a special kind of antenna designed to focus on a specific broadcast source [3]. The standard dish consists of a parabolic (bowl-shaped) surface and a central feed horn. The dish on the receiving end can't transmit information; it can only receive it. The central element in the feed horn is the low noise block down converter, or LNB which amplifies the radio signal bouncing off the dish and filters out the noise (radio signals not carrying programming), it then passes the amplified, filtered signal to the satellite receiver inside the viewer's house.

This signal can be affected by activities that occur in the atmosphere which result in signal fluctuation. These 
fluctuation in signals are as a result of many factors which are being contained in the atmosphere, such factors are the atmospheric parameters, atmospheric gas, and the troposphere also have an effect on signal strength. Attenuation due to atmospheric gases is mainly due to oxygen and water vapor absorption. Oxygen possesses a paramount magnetic moment and because of interaction of this moment with the magnetic field of wave, absorption of wave energy takes place [4].

Specifically, it is known that adverse weather can cause signal losses. Basically, weather is the state or condition of the atmosphere, describing for example the degree to which it is hot or cold, wet or dry, calm or stormy, clear or cloudy. It involves atmospheric parameters such as temperature, humidity, precipitation, air pressure, wind and cloud cover. Signal path loss is an essential factor in the plan of any radio communication system [5]. Signal path losses are the degradation in signal strength as it travels through a particular region or medium [6]. Changes in the weather condition affect the quality of the satellite television signal reception though this occurs rarely, and lasts only a short period. For the majority of users, it is heavy rain, high winds, snow, ice and heavy fog that can attenuate signal enough to result In noticeable degradation of image signal but in Africa and Nigeria to be preside we experience little or no snow, ice and fog. The level of concern about the possibility of signal degradation/loss in a particular area depends on the following factors: Regional yearly rainfall figure, Location of the satellite, Height of the satellite above the horizon, Wind speed.

The Rainfall pattern and wind speed experienced in a place has much effect on satellite dishes and can cause attenuation of signal. During a rainstorm, the raindrops can weaken or absorb the signal on its way to a satellite dish. Rain can also cause signal scattering as electromagnetic waves refract and diffract around raindrops on the surface of the dish. The most common causes of signal attenuations by rain are scattering and absorption. Scattering is the process by which signals interact with transmission in a way that causes the signal to travel in direction different from the original direction. The interaction between the radio frequency (RF) energy and particles causes scattering [7]. Scattering also occurs when signal travelling in the atmosphere encounters rain droplet and are scattered in different directions [8]. The scattering effects are mostly noticed in TV set as interference. Sometimes the received signal on the TV disappears and after sometimes the qualities of the pictures and the audio will be badly affected. Moreover, scattering can occur inform of refraction and diffraction. This affects the signals by reducing the strength before it reaches the receiving end [9]. Rain attenuation due to absorption occurs when radio wave travelling in the atmosphere strikes a rain droplet, part or all the energy of the signal is converted to heat and is absorbed by it. Absorption by molecular resonance in the atmosphere is a major factor affecting radio propagation. If the rain is accompanying by high winds, as a result of this the dish may be out of alignment with the satellite which affects the signal.
This effect of rain on signal has drawn considerable attention in Nigeria as this occur at frequencies above $10 \mathrm{GHz}$ and the prevailing influence in the tropical and subtropical regions is evidenced in recent contributions, especially from locations where rainfall is characterized with high and rapidly varying intensities [10]. Rain effects are dependent on frequency, rain rate, drop size distribution and drop shape, which are determined by the type of rain [11]. Recent studies have however shown that the transmitted radio signals may go through spatial and temporal changes due to variations in the atmospheric conditions as well as other environmental factors [12]. These conditions vary with changes in height, geographical location, and even with changes in time of the day as well as seasons of the year.

This research evaluates the effect of weather parameters (temperature, pressure, humidity and wind speed) on satellite dishes and how it affects the quality of reception it gives using the data obtained from the Automatic Weather Station and Signal Strength Meter in Enugu State University of Science and Technology (ESUT) for a period of nine months.

\section{Source of Data}

The data used for this work was downloaded from Automated Weather Station and Signal Strength Meter (AWSSSM) in the Department of Industrial Physics Enugu State University of Science and Technology.

\section{Methods}

We downloaded data from a signal receiving system, which was set up by connecting a high gain ultra-high frequency antenna and spectrum analyzer. The research was carried out in the AWSSSM in ESUT a residential/schooling area (Agbani) within the Enugu metropolis in Enugu State, Nigeria. Signal strengths measurements were obtained every two minutes at the residential area and simultaneously for period of nine months. The weather components are temperature, pressure, relative humidity and wind speed were recorded to probe the impact of the weather components on signal strength, and satellite dishes. Computation of signal strength, temperature, humidity, pressure was considered in each case, statistical analysis using excel program will be carried out in order to ascertain the level of relationship between signal strength, atmospheric temperature, relative humidity, pressure and wind speed. The measurement was made using satellite dish which is focused on ASTRA1R $15.2^{\circ} \mathrm{E}$ located at the geostationary orbit or Clark orbit. The dish concentrates the signal at Low Noise Block (LNB). The LNB is a universal type receiving $10.7 \mathrm{GHz}-12.6 \mathrm{GHz}(\mathrm{Ku}$ Band), it then down converts it to signal between $950 \mathrm{MHZ}-$ $2150 \mathrm{MHz}$. The major Reason for the conversion is that the $10.7 \mathrm{GHz}-12.6 \mathrm{GHz}$ signal cannot be transmitted through coaxial cable (because of attenuation). Therefore, a lower frequency was chosen for the transmission into the internal unit via cable. 


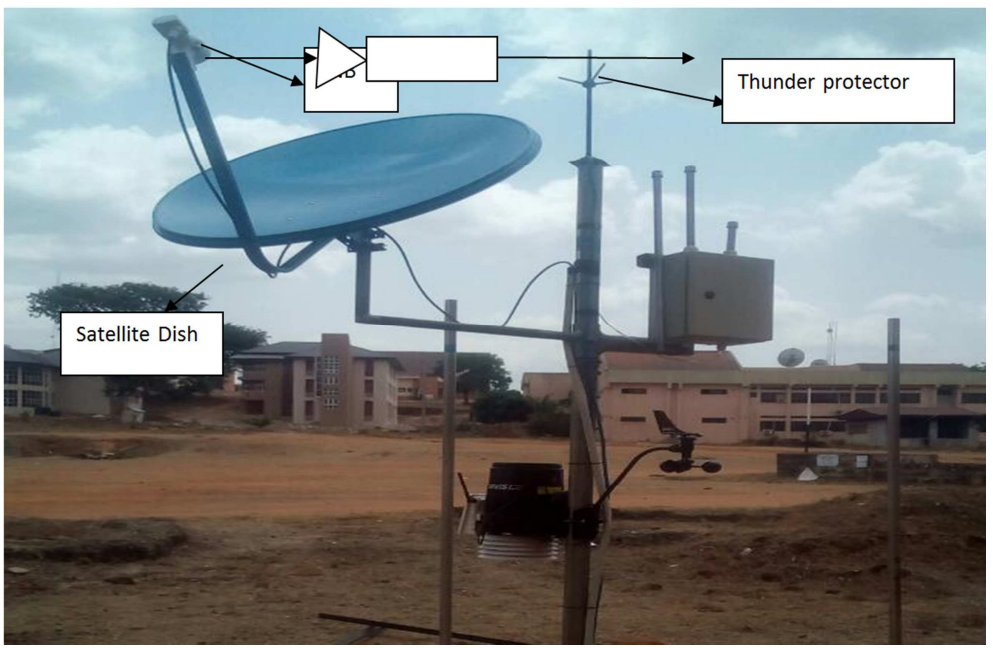

Figure 1. Showing the pictorial representation of the AWSSSM in ESUT.

\section{Results}

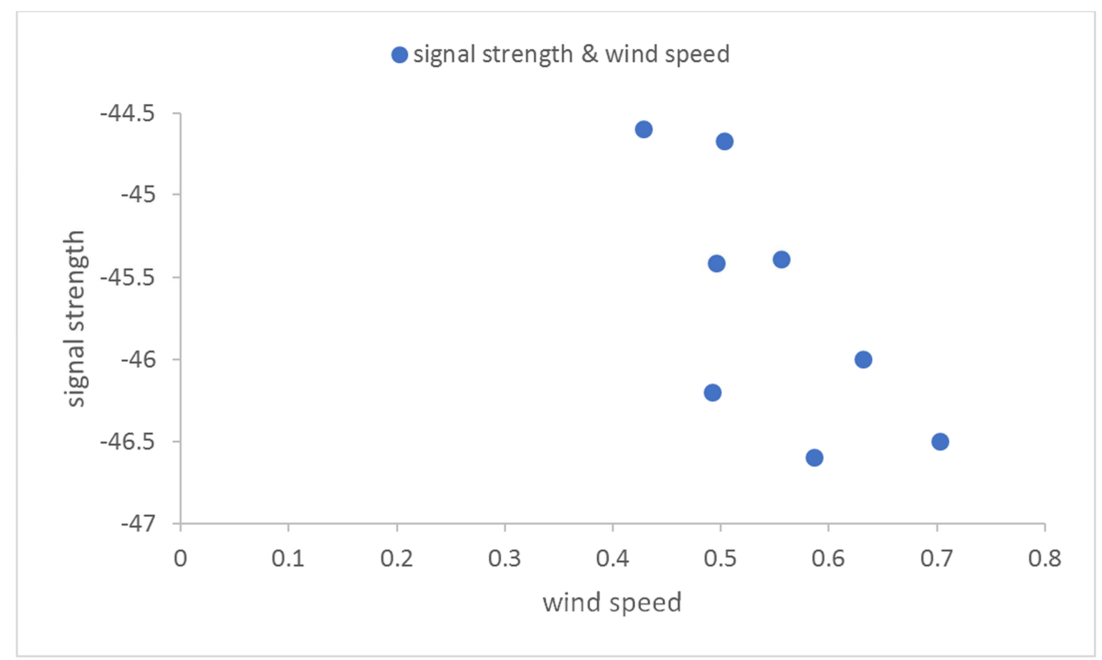

Figure 2. Showing the relationship between signal strength and wind speed from January to August.

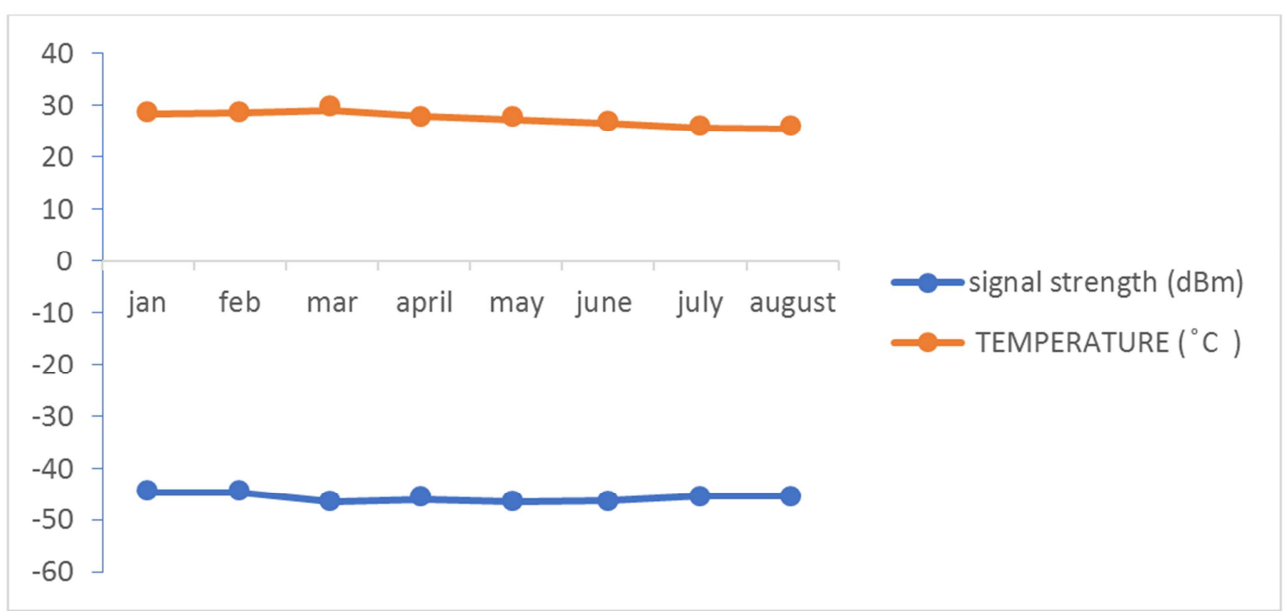

Figure 3. Showing the relationship between signal strength and temperature from January to August. 


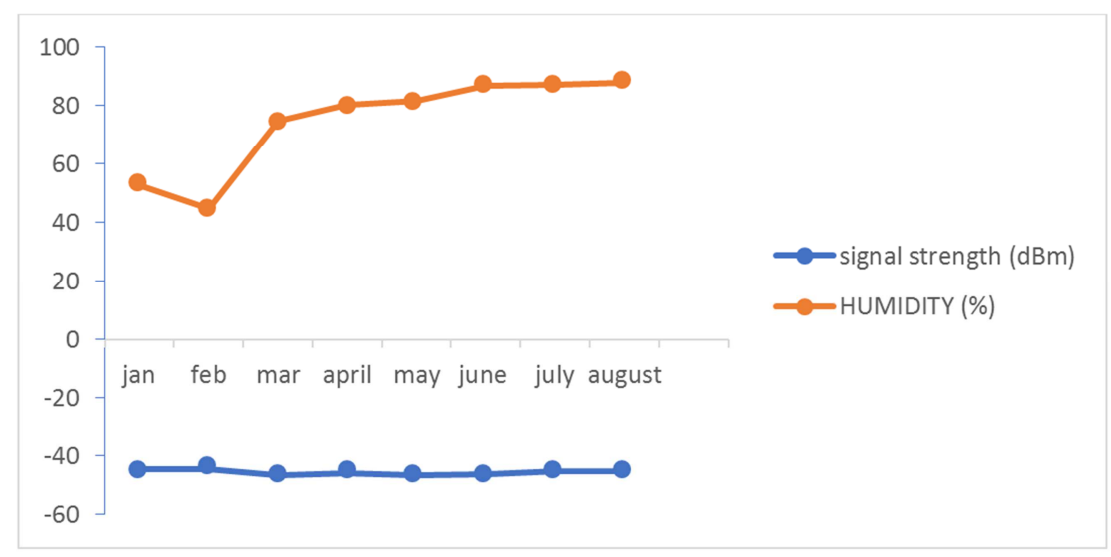

Figure 4. Showing the relationship between signal strength and humidity from January to August.

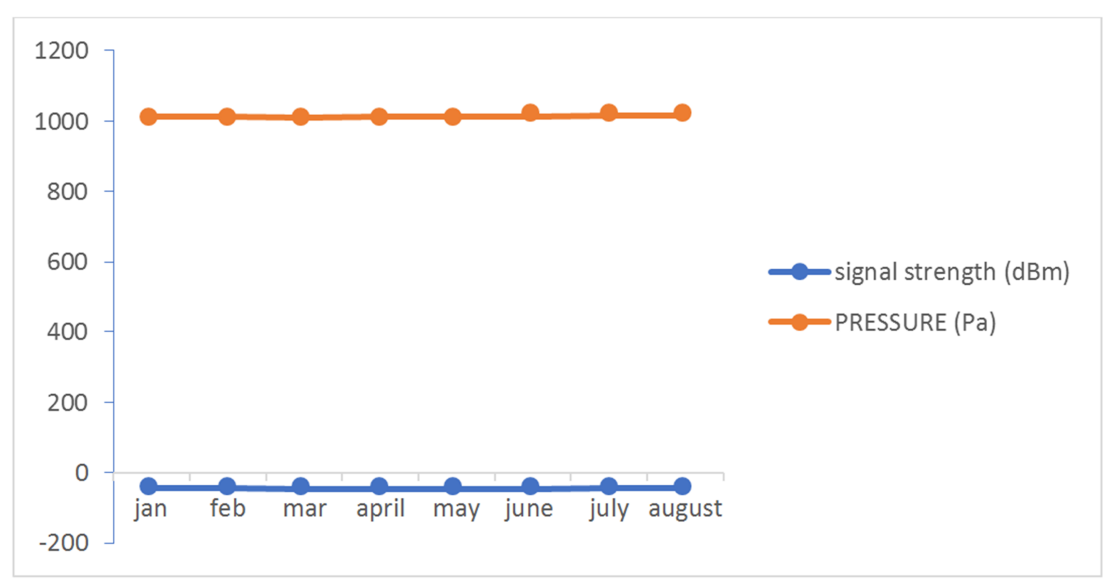

Figure 5. Showing the relationship between signal strength and pressure from January to August.

\section{Discussion}

This section contains the detail explanation on the effect of weather parameters (temperature, humidity, pressure including wind speed and direction) on signal strength of satellites dishes in Enugu metropolis for nine months (January-September 2019).

From the graph plotted there is an obvious relationship between temperature and signal strength, which shows that even a slight rise in temperature brought about an overwhelming fall in signal strength and vice versa. For the month of January to March, there is a noticeable increase and decrease in temperature but it effect is not much on signal strength but in April there is a strong fluctuation between signal strength which consist of a sharp increase and decrease due to slight variation in temperature while for other months (May to September) there are sharp variation of temperature leading to slight fluctuation of signal strength with sharp decrease from time to time.

Another potential factor affecting signal strength is humidity. This is due to the water vapor in the atmosphere, as the signal travels in form of electromagnetic wave, it can easily be absorbed, reflected, scattered and diffracted etc. by water vapor in the air as it propagates. In the month of January to march, there is variation in humidity which causes slight decrease and increase in signal strength, in the month of April there are sharp variation in humidity which causes a sharp decrease and increase of signal strength, therefore the fluctuation of signal strength caused by humidity in this month is not negligible. While in May a slight rise and fall of humidity brought about rapid fluctuation of signal strength at the beginning of the month and a sharp fall at the ending of the month, but in June and July there is a sharp increase and a slight decrease of signal strength. The month of August shows a very sharp fall and slight fluctuation of signal strength, we found out that the reason behind the sudden change can be attributed to August break which means there is not much rainfall and trend continues to the month of September. The signal strength decreased with increase in humidity and vice versa. Mathematically, the signal strength is inversely proportional to humidity, provided atmospheric pressure and temperature and wind speed and direction are observed constant.

At higher pressures, the atmospheric oxygen causes considerable attenuation and a small-time delay in the received signal. From the month of January to March there is little variation in atmospheric pressure but little effect on signal strength. From April-July, there is a gradual rise and fall of atmospheric pressure, which was due to high pressure in the atmosphere which brings about high water content in the air, which condenses and fall as rain, bringing about the absorption and scattering of signal by rain causing a strong signal fluctuation during those months. In August, the pressure rises 
and fall sparingly because of the august break in rainfall.

Results obtained from this study also shows that wind speed affected signal strength in contrast to some past research which said that wind speed has no impact on signal strength [14]. In figure 2 above, it was observed that there is a noticeable fluctuation of signal strength which is as a result of increase and decrease in wind speed. Radio wave which has shorter wavelength is more attenuated by absorption and scattering from dust and sand (which is triggered by wind) in the radio path resulting in loss of signal energy. Wind speed can have a direct impact on a satellite dish and this occurs when there is relative increase in wind speed which can move the satellite dish out of alignment and cause service disruption and no signal can be received.

\section{Conclusion}

It was observed that the atmospheric temperature, humidity, pressure and wind speed are mathematically inversely related. If $\mathrm{S}, \mathrm{P}, \mathrm{T}$ and $\mathrm{H}$ symbolize signal strength, atmospheric pressure, atmospheric temperature and relative humidity respectively, it can be postulated that

$$
\begin{aligned}
& \mathrm{S} \alpha \frac{1}{\mathrm{PTH}} \\
& \mathrm{S}=\frac{\mathrm{K}}{\mathrm{PTH}}
\end{aligned}
$$

In all the nine months analyzed we found out that during raining season, atmospheric parameters (temperature, pressure, and humidity) impact is highly felt on signal strength unlike the dry seasons and this explained why series of network fluctuations occur more during raining seasons. And also high wind speed can have effect on satellite dishes because wind can bend or move a dish out of alignment with incoming signal or to the point of service disruption causing temporary outages as the signal gets bent. These effects are been experience in TV, satellites, telecommunications network etc. from the analysis we found out that the stronger the received signal, the more immune atmospheric parameters, so weather affects weaker systems more severely which is in line with other authors [13].

The correlation of the signal strength and atmospheric temperature, pressure and humidity were $\mathrm{r}=-0.93,-0.97$ and 0.92 and thus the equation $\mathrm{S}=\frac{\mathrm{k}}{\mathrm{TPH}}$ was postulated, where $\mathrm{S}, \mathrm{T}$, $\mathrm{P}, \mathrm{H}$ and $\mathrm{K}$ are Signal strength, Atmospheric temperature, Atmospheric pressure, Relative humidity are constant which is as a result of constant change in weather condition. We observed that it is difficult to get a good network signal on satellite dishes when it is raining and when there is relative increase in wind speed due to the fact that wind speed can move the satellite dish out of alignment and cause service disruption and no signal can be received. And to support the above postulation, it was also observed from the data gotten in the month of June and July which is the peak of rainy season that bad signals are most frequently noticed than that of January and February which is the peak of dry seasons.

\section{Acknowledgements}

We Sincerely Thank the Department of Industrial Physics, Enugu State University of Science and Technology for Allowing Us Use The Data Obtained From There Automated Weather Station To Carry Out This Research which was denoted to them by Tertiary Education Fund (TETFUND), Nigeria through the National Research Fund (NRF) grant.

\section{References}

[1] Martin, Donald; Anderson, Paul; Bartamian, Lucy (2007) communications satellites.

[2] Hoffmann, (2007): Academic's Dictionary of Mass Communication. New Delhi, Academic (INDIA) Publishers, India.

[3] Evangelista, Benny (2003). Satellite television on the move/new technology to make dish receiver small enough to fit atop a car.

[4] Martin hall, (2013) Les Barclay: propagation of radio waves, 2nd edition; the institute of electrical engineering, London UK, meteorological society.

[5] Wayne T. (2001). Electronic communication systems. Fundamentals through advanced. New Jersey: practice. Hall. 39.

[6] Ian P (ED) (2015) Radio signal path loss in radio electronics, radio propagation and the atmosphere.

[7] Tom W. and Waimba (1995). Narrow Band 10GHz and some observation. New England VHF conference, ARRL Publication.

[8] Friedeu R (2009). Wireless Communication. Microsoft Encarta Glossary of meteorology.

[9] Demetrius and Kenneth (1969) Ionospheric radio. Definition; broadcasting satellite service.

[10] Ajayi, G. O. (1989). Physics of the tropospheric radio propagation. A proceeding of the ICTP College on Theoretical and Experimental Radio Propagation Trimester, Italy, 6-24.

[11] Adami D., Marchese M and Ronga L. S. (2000). A Quality Service guarantee in IP Satellite environment, experimental experience in the CNIT-ASI project, integration of multimedia services on heterogonous satellite networks. Proc. GLOBECOM. Sam Francisco.

[12] Gunashekar. S. D, D. R. Siddleande, M Warrington, (2006). Trans Horizon Radio Wave Propagation Due to Evaporation Ducting. The Effect of Tropospheric Weather Conditions on Vhf and Uhf Radio Paths Over Sea. "Resonance Journal of Science Education, Indian Academy of Science, Bangalore, India. Volume 11, Number I.

[13] Communication Satellite "Wikipedia" (2016). Retrieved from: https//en.wikipedia.

[14] Holland. M, R. Aureus and W. Heinzelman, (2006). Experimental investigation of radio performance in wireless sensor networks. Networks. Wimesh, 2nd Edition EEE workshop. 